\title{
Seguimento farmacoterapêutico direcionado aos animais domésticos: seria viável?
}

\author{
Pharmacotherapeutic Follow-up directed to domestic animals: \\ would it be feasible?
}

Recebido em: 07/08/2014

Aceito em: $13 / 09 / 2014$

\begin{abstract}
Maria Aparecida NICOLETTI ${ }^{1,2}$, André Rinaldi FUKUSHIMA ${ }^{2,3,4}$
${ }^{1}$ Universidade de São Paulo - Faculdade de Ciências Farmacêuticas. Avenida Professor Lineu Prestes, 580 - Departamento de Farmácia. CEP 05508-900 São Paulo, SP. Brasil.

${ }^{2}$ Universidade de Guarulhos Pca. Tereza Cristina, $n^{\circ} 88$, Centro Guarulhos / SP CEP:

07023-070 3 Universidade São Judas Tadeu Unidade Mooca - R. Taquari, 546 - Mooca

- São Paulo/SP - CEP 03166-000 ${ }^{4}$ Departamento de Patologia - FMVZ-USP Faculdade de Veterinária e Zootecnia Universidade de São Paulo (USP) Av. Prof. Dr. Orlando Marques de Paiva, 87 CEP 05508270 - Cidade Universitária São Paulo/SP - Brazil E-mail:fukushima@usp.br
\end{abstract}

\section{ABSTRACT}

The overall performance in the area of human health has directed its focus to patient care, taking the medicine as the background. In this sense, the human pharmacotherapeutic follow-up (which is part of the Pharmaceutical Care) has been a great tool to aid the detection of Drug Related Problems (DRP) so they can be avoided or identified in order to prevent / minimize the emergence of Drug Negative Outcomes (DNO). Considering the need of therapeutic success as a result to obtain in health care, a reflection on the possibility of a follow-up model (Dáder modified) to be adapted and applied to the pharmacotherapeutic monitoring animal area, particularly, in domestic animals with chronic use of medications is presented.

Keywords: pharmacotherapeutic follow-up, patient safety, domesticated animal.

\section{RESUMO}

A atuação global na área da saúde humana tem direcionado seu foco ao cuidado do paciente, tendo o medicamento como segundo plano. Neste sentido, em humanos o seguimento farmacoterapêutico (que faz parte da Atenção Farmacêutica) vem sendo uma ferramenta de grande auxílio para a detecção de Problemas Relacionados a Medicamentos (PRM) para que possam ser evitados ou identificados visando impedir/minimizar o aparecimento de Resultados Negativos relacionados a Medicamentos (RNM). Considerando a necessidade do sucesso terapêutico como o resultado a ser obtido no cuidado à saúde, o presente trabalho apresenta uma reflexão sobre a possibilidade do modelo de seguimento farmacoterapêutico (Dáder modificado) ser adaptado e aplicado à área animal, particularmente, em animais domésticos que fazem uso crônico de medicamentos.

Palavras Chave: segurança do paciente, seguimento farmacoterapêutico, animais domésticos 


\section{INTRODUÇÃO}

O mundo globalizado estabelece foco diferenciado em relação ao papel de gestores e profissionais clínicos em razão de estarem envolvidos dois aspectos de importância fundamental: a questão financeira e o resultado clínico efetivo. Entretanto, ao se introduzir no universo de conceitos utilizados para a avaliação de desempenho, cada um dos aspectos mencionados anteriormente revela características distintas para a execução plena das atribuições (1).

Muito embora atividades relacionadas à gestão e à clínica apresentem dimensões diferenciadas há necessidade de um foco comum que é alcançar o bem estar do paciente e, assim, entende-se que o animal poderá ser, também, introduzido neste foco.

A terapia medicamentosa assume uma participação decisiva no desfecho terapêutico do animal para a recuperação da saúde, e uma condição de equilíbrio que permita a ele manter seu estado de saúde, ou mesmo uma condição de menor desconforto em algumas situações, quando a recuperação da saúde não se apresenta mais como uma alternativa possível.

Traçando um paralelo com a saúde humana, as práticas em serviços de saúde, com diversas linhas de entendimento, estão atualmente sendo muito discutidas considerando a necessidade de promoção de qualidade de vida das pessoas, em particular, no que diz respeito ao uso racional de medicamentos (2), e nas quais podem ser encontradas semelhanças com a saúde animal (3).

Alguns conceitos na área da saúde humana, mais particularmente ligados à Assistência Farmacêutica no Sistema Único de Saúde, estão sendo discutidos para que a prática das ações envolvidas se torne uma atividade que proporcione melhoria tanto para o cidadão quanto para o sistema de saúde pública, com a diminuição de gastos em saúde e melhoria de qualidade de vida dos indivíduos (4).

Dentro da Assistência Farmacêutica, a prática denominada "Atenção Farmacêutica" (Pharmaceutical Care), em saúde humana, está relacionada a uma proposta de seguimento farmacoterapêutico que o profissional farmacêutico desenvolve junto aos pacientes com a finalidade de aumentar a eficácia terapêutica, a segurança no uso do medicamento, minimizar o aparecimento das reações adversas de medicamentos (RAM), bem como detectar outros possíveis problemas decorrentes do uso do seu uso (5).

A atenção farmacêutica é uma filosofia profissional que pode ser incorporada por todos os profissionais farmacêuticos, independentemente do espaço físico de onde atuam. Representa a decisão entre ações importantes a serem assumidas como a utilização de método sistemático e racional de tomada de decisão sobre medicamentos, e o cuidado ao paciente (6).

O conceito de Atenção Farmacêutica, aceito mundialmente, é estabelecido como "a provisão responsável da farmacoterapia com o propósito de alcançar resultados definidos que melhorem a qualidade de vida do paciente" (7). Entretanto, no Brasil, somente a partir de 2002 ocorreu a intensificação das discussões a respeito desta prática (8).

As doenças crônicas, quando presentes, são objeto de preocupação porque poderão comprometer a qualidade de vida, quer humana quer animal, considerando que mais do que um medicamento será administrado concomitantemente facilitando, assim, a ocorrência de interações medicamentosas, que poderão fragilizar o processo de recuperação da saúde.

Neste sentido, a prática da atenção farmacêutica tem sido aplicada com muito sucesso na área da saúde humana por meio do seguimento farmacoterapêutico do paciente porque o foco principal em saúde pública atual é o tratamento humanizado do paciente, e não mais o medicamento (9). Normalmente, é entendida como uma prática que tem uma interface multiprofissional considerando que diversas áreas devem ser levadas em consideração como nutrição, fisioterapia, entre outras, para atuarem no restabelecimento e/ou manutenção da saúde $(10,11,12)$.

O sucesso da terapia medicamentosa requer uma análise detalhada do desenvolvimento do processo, para que intervenções possam ser realizadas, por meio da proposição de seguimento farmacoterapêutico e que poderá ser estabelecido com a utilização de vários modelos disponíveis na literatura.

Estes modelos permitem uma avaliação muito clara de possíveis problemas relacionados a medicamentos (PRM) para que sejam evitados os resultados negativos relacionados a medicamentos (RNM), termos atualmente muito empregados na avaliação do regime terapêutico proposto. Define-se como suspeita de RNM, a situação em que o paciente apresenta risco de sofrer problema de saúde associado ao uso de medicamentos, geralmente pela existência de um ou mais PRM, o que pode ser considerado como fator de risco no aparecimento de RNM (13).

Considerando o medicamento como uma das ferramentas utilizadas para a recuperação da saúde, o entendimento dos fatores relacionados à sua eficácia, segurança e qualidade tem uma grande influência no sucesso da terapia medicamentosa e, portanto, o seguimento far- 
macoterapêutico pode se tornar parte do processo de recuperação de saúde animal, tal qual o faz para humanos.

O principal aspecto da prática do seguimento farmacoterapêutico em animais seria o de impedir ou minimizar os problemas relacionados a medicamentos.

Para uma proposta de terapêutica correta e de seu seguimento é necessário que a equipe de saúde envolvida considere a eficácia do fármaco, a efetividade da farmacoterapia selecionada e a eficiência de todo processo de tratamento. Deve ser entendido que a eficácia é o melhor resultado em condições específicas, a efetividade como o resultado terapêutico produzido nas condições reais de utilização e a eficiência como a obtenção do maior benefício com o menor custo. Correr e cols. (2011) evidenciaram o modelo lógico conceitual da Assistência Farmacêutica integrada ao processo de cuidado em Saúde, para uso humana (14). Quanto à gestão clínica do medicamento, o presente trabalho tem como objetivo mostrar que possível o estabelecimento de analogia com a prática veterinária é possível. No caso da aplicação de Gestão Clínica do Medicamento à área veterinária, a identificação de problemas que apresentem diversos vieses, direciona às intervenções necessárias para o sucesso do desfecho terapêutico.

O animal é um ser complexo, e sua comunicação com os seres humanos ocorre por meio de sinais, que nem sempre são bem interpretados, implicando em uma problemática em relação à aplicação, na íntegra, do protocolo da Gestão Clínica do Medicamento em humanos, que deverá obviamente ser adaptado para os animais. Políticas públicas com a finalidade de tratamento farmacológico dos animais ainda são escassas no Brasil.

De modo geral, a saúde dos animais está intrinsecamente ligada à saúde humana quando se trata de animais para consumo, além daqueles contaminados com doenças negligenciadas que se tornam reservatórios de parasitas, como, por exemplo, diversos tipos de Leishmaniose, verminoses, entre outras de elevada incidência em nosso país. Portanto, no que tange à área de saúde pública, a saúde animal pode ser considerada um parâmetro diretamente relacionado à saúde humana, quando se pensa em determinadas situações.

Os métodos disponíveis para uso no seguimento farmacoterapêutico de humanos são vários e os mais utilizados são Dáder (que leva o nome de sua autora), TOM (Therapeutic Oncologic Monitoring), PW (Pharmacist's Workup), e SOAP (Subjetivo, Objetivo, Avaliação e Plano). De modo geral, os métodos têm particularidades e propostas diferenciadas; entretanto, de modo simplista pode ser estabelecido que vários tipos de informação estejam presentes nos formulários correspondentes, como: a anamnese do paciente, o estudo de alternativas terapêuticas possíveis e viáveis, o objetivo terapêutico proposto, a estruturação do plano de monitoramento para alcançar o objetivo terapêutico traçado, a análise do uso dos medicamentos e a identificação de possíveis problemas relacionados a medicamentos ou a detecção de resultados negativos relacionados a medicamentos; ainda, reações adversas ou falha terapêutica para novas intervenções, estabelecimento de datas para a continuidade do seguimento, entre outras que são necessárias, dependendo do método adotado $(15,16)$.

Os formulários podem ser estruturados/adaptados considerando as linhas adotadas de prática clínica que favoreçam o seguimento farmacoterapêutico do animal e para aqueles que apresentam necessidade da utilização de vários medicamentos é verificada uma grande probabilidade de interações medicamentosas que, certamente, afetam o resultado final de restabelecimento da saúde animal. Segundo Oga (2008) conforme os mecanismos envolvidos, as interações podem ser físico-químicas, farmacocinéticas e farmacodinâmicas e, em cada uma delas, muitos fatores estão envolvidos (17).

O resultado de uma intervenção medicamentosa não pode ser visto de forma isolada, considerando todos inúmeros fatores que poderão incidir na saúde animal, em níveis diferentes de complexidade. Não poderão ser esquecidos os atores envolvidos neste processo: o animal, o proprietário e o veterinário. Neste sentido, o farmacêutico na prática do seguimento farmacoterapêutico do animal será o outro ator que estará diretamente ligado à identificação dos problemas relacionados a medicamentos (por exemplo, desvio de qualidade, falta de efetividade terapêutica, reação adversa ou toxicidade, interações com medicamentos, alimentos, interferência em exames laboratoriais, etc.) sejam eles reais ou potenciais, ou seja, resolver os problemas reais e prevenir os problemas potenciais.

A prática de atenção farmacêutica na área de saúde animal é certamente uma ferramenta inovadora que poderá colaborar na recuperação da saúde de uma maneira facilitada por meio de um seguimento farmacoterapêutico que possibilita a mensuração dos resultados obtidos, particularmente, do desfecho terapêutico. Além dos aspectos anteriormente mencionados, a prática descrita possibilita a análise de custo-efetividade, ou seja, permite comparar intervenções que tenham em comum um mesmo desfecho em saúde (15).

Os modelos em humanos podem servir como base para a proposição de um modelo de atenção que se aplique ao animal, muito embor seja necessário realizar uma divisão mais profunda que a humana, como segmentos farmacoterapêuticos individualizados por espécie ani- 
mal e patologia, pois, existe uma enorme variação fisiológica e metabólica interespécie, em relação à conduta alimentar dos animais (3).

A área de neurocomportamento nos fornece dados importantes e estabelece praticamente uma comunicação não verbal entre o homem e o animal. Portanto, a observação do comportamento animal, bem como a aplicação de testes comportamentais nos mesmos poderia fornecer dados importantes com finalidade de verificar a progressão do tratamento farmacológico no animal (18), de maneira não invasiva, e com sensibilidade suficiente para detectar PRM.

O foco na segurança do paciente pode ser caracterizado pela preocupação com a magnitude da ocorrência de eventos adversos (EA), ou seja, com lesões ou danos ao paciente ocasionados pelo cuidado de saúde (19). Na verdade, o que se propõe com o seguimento farmaco- terapêutico é o estudo de efetividade terapêutica direcionado a animais, que analisa o resultado terapêutico produzido pelo(s) medicamento(s) nas condições reais de utilização. A área denominada de Farmacovigilância, também conhecida como "ensaios pós-comercialização" ou "Estudos Clínicos Fase IV", tem como um de seus principais objetivos a detecção precoce de reações adversas, especialmente as desconhecidas (20). Tal fato justifica um seguimento contínuo para a avaliação de sua segurança e efetividade terapêutica quando consideramos a administração do medicamento inovador ou novo, lançado para comercialização.

A Figura 1 representa uma proposta de de seguimento farmacoterapêutico a ser aplicado em animais, principalmente àqueles que sejam usuários crônicos de medicamentos, inclusive medicamentos inovadores na área veterinária, com base no Método Dáder (21).

Figura 1. Proposta de fluxograma para seguimento farmacoterapêutico em animais, com base no Método Dáder (21).

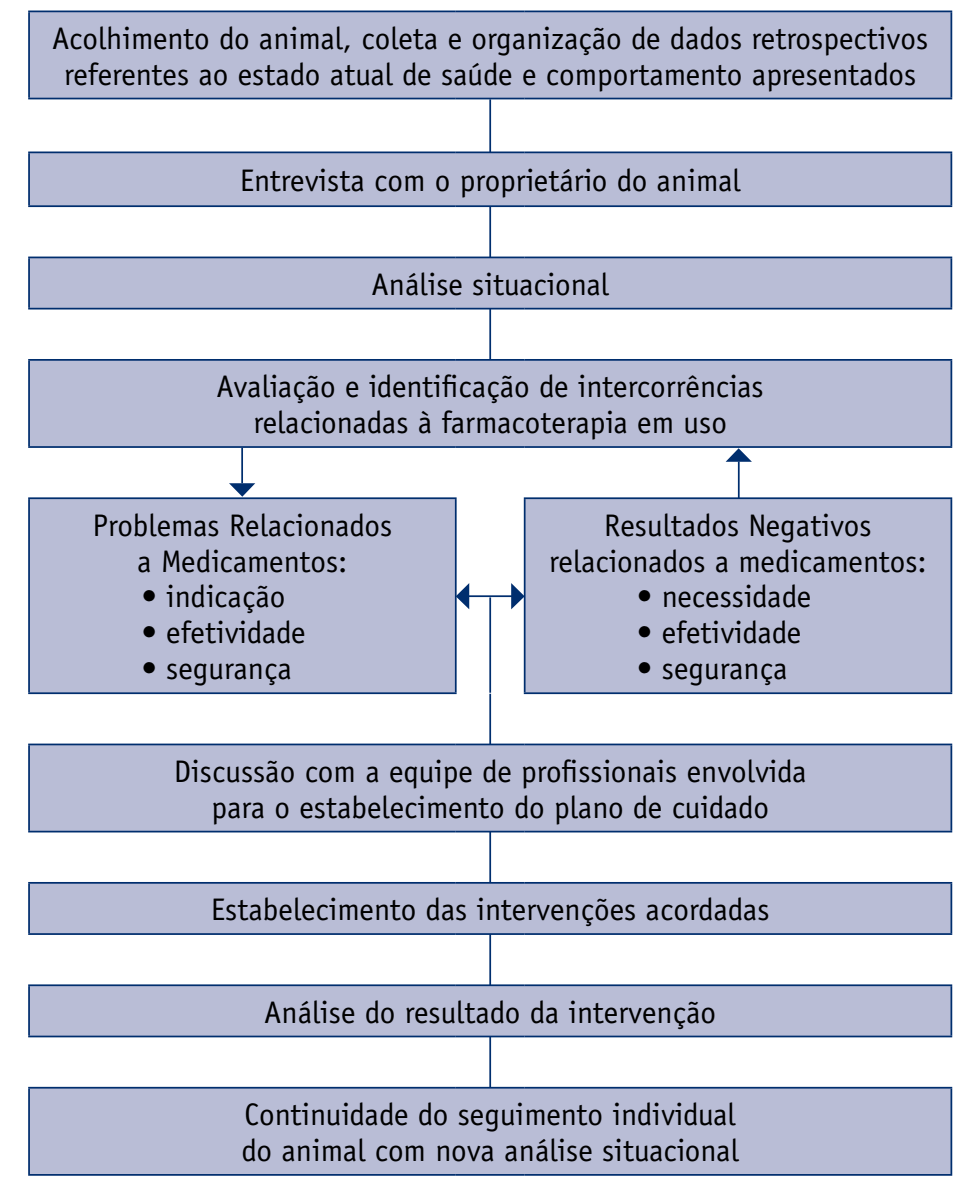

Por esse modelo, poderão ser avaliados os desfechos clínicos (primordiais, intermediários e substitutos), ou seja, mudanças observadas no estado de saúde do animal decorrentes das intervenções realizadas, e que capacitarão o alcance das metas terapêuticas propostas, mensuráveis por meio dos parâmetros clínicos e/ou la- 
boratoriais associados a prazos definidos, para o alcance dos resultados.

Dessa forma, a prática de seguimento terapêutico em animais poderá ser uma ferramenta de valor para o acompanhamento daqueles que apresentam doenças crônicas ou de medicamentos veterinários inovadores considerando as possíveis interações indesejáveis e eventos adversos que poderão ocorrer, além da ineficácia terapêutica. Certas morbidades necessitam de ajuste de dose além de esquemas posológicos bem específicos considerando o momento de saúde que o animal esteja vivendo, e o desfecho terapêutico esperado poderá ser comprometido se as inúmeras variáveis envolvidas no processo não forem adequadamente consideradas. Adiciona-se, também, o benefício de bancos de dados estruturados que poderão gerar algoritmos de condutas facilitando, assim, o processo de condução terapêutica no restabelecimento ou manutenção de qualidade de vida do animal.

\section{REFERÊNCIAS}

1. UFSC. Universidade Federal de Santa Catarina. Módulo transversal 1: gestão da assistência farmacêutica. Universidade Federal de Santa Catarina, Universidade Aberta do SUS. Florianópolis: Ed. da UFSC, 2012. Disponível em: $<$ www.unasus.ufsc.br $>$.

2. BRASIL. Conselho Nacional de Secretários de Saúde. Assistência Farmacêutica no SUS. Brasília, D.F.: CONASS. 2011.

3. Spinosa HS, Gorniak SL, Bernardi MM, organizadores. Farmacologia aplicada à medicina veterinária. $5^{\mathrm{a}}$. Ed. Rio de Janeiro: Guanabara Koogan, 2011.

4. Castro MS, Fuchs FD, Ferreira, MBC. Reflexão sobre a prática e o ensino do seguimento farmacoterapêutico de pacientes. In: Storpirtis S, Mori ALPM, Yochy A, Ribeiro E, Porta, V. Farmácia clínica e atenção farmacêutica. Rio de Janeiro: Guanabara Koogan. 2008. p.360-66.

5. Portal Educação e Sites Associados. Programa de Educação Continuada a Distância. Curso de Atenção Farmacêutica. Módulo 1. 2012. 1: 1-10.

6. Cipolle RLJ, Strand LM, Morley PC. Pharmaceutical care practice: the clinician's guide. 2nd. ed. New York: McGraw-Hill. 2004.

7. Hepler C, Strand L. Opportunities and responsibilities in pharmaceutical care. Am J Hosp Pharm. 1990. 47: 533543.

8. OPAS. Organização Pan-Americana da Saúde. Consenso brasileiro de Atenção Farmacêutica: proposta. Brasília (DF). 2002.

9. Goulart BNG, Chiari BM. Humanização das práticas do profissional de saúde - contribuições para reflexão. Ciênc Saúde Colet. 2010. 15(1): 225-268 http://dx.doi. org/10.1590/S1413-8123201000010003.

10. Cordeiro BC, Leite $\mathrm{SN}$. O farmacêutico na atenção à saúde. Itajaí: Universidade do Vale de Itajaí, 2005. 189 p.

11. Witzel MDRF. Aspectos conceituais e filosóficos da Assistência Farmacêutica, Farmácia Clínica e Atenção Farmacêutica. In: Storpirtis S. et al. (Eds.) Farmácia clínica e atenção farmacêutica, Rio de Janeiro: Guanabara Koogan, 2008. p. $336-348$.
12. Ramalho de Oliveira D. Atenção farmacêutica: da filosofia ao gerenciamento da terapia medicamentosa. São Paulo: RCN, 2011. 328p.

13. Comité de Consenso. Tercer Consenso de Granada sobre Problemas Relacionados com Medicamentos (PRM) y Resultados Negativos asociados a la medicación. Ars Pharm. 2007. 48(1): 5-17.

14. Correr CJM, Otuki M, Soler, O. Assistência farmacêutica integrada ao processo de cuidado em saúde: gestão clínica do medicamento [on line]. Rev Pan-Amaz Saúde. 2011. 2(3):41-49.

15. Kernick, D, editor. Getting health economics into practice. U.K: Radcliffe Medical Press. 2002.

16. Marasciulo AC, Cordeiro BC, Storb BH, Blatt CR, Farias MR. Avaliação econômica em saúde. In: Universidade Federal de Santa Catarina. Conteúdos optativos [Recurso eletrônico]. Universidade Federal de Santa Catarina/ Universidade Aberta do SUS. Florianópolis: Ed. da UFSC. 2011.

17. Oga S. Interações medicamentosas. In: Storpirtis S, Mori ALPM, Yochy A, Ribeiro E, Porta V. Farmácia clínica e atenção farmacêutica. Rio de Janeiro: Guanabara Koogan. 2008. p.78-85.

18. Ender L, Massoco, CO, Spinosa HS, Gorniak SL, Bernardi MM. Efeitos da administração aguda e prolongada do Catnip (Nepeta cataria) a camundongos em modelos compartimentais de depressão e ansiedade. Rev Inst Ciênc Saúde. 1998. 16(1): 7-11 http://dx.doi.org/10.3922/j. psns.2010.2.015.

19. Reis CT, Martins M, Laguardia J. A segurança do paciente como dimensão da qualidade do cuidado de saúde - um olhar sobre a literatura. Ciên Saúde Colet. 2013. 18(7): 2029-2036 http://dx.doi.org/10.1590/S1413$\underline{81232013000700018}$.

20. Romano-Lieber NS. Princípios de farmacoepidemiologia. In: Storpirtis S, Mori ALPM, Yochy A, Ribeiro E, Porta V. Farmácia clínica e atenção farmacêutica. Rio de Janeiro: Guanabara Koogan. 2008. p.37-45.

21. Hernandez DS, Castro MMS, Dáder, MJF. Método Dáder - Manual de seguimento farmacoterapêutico 2009. $3^{\text {a }}$. ed. Lisboa: Edições Universitárias Lusófonas. 2010. 